\title{
H Consensus Control of Multi-agent Systems with Input Delay and Directed Topology
}

DOI:

10.1049/iet-cta.2015.0945

\section{Document Version}

Accepted author manuscript

Link to publication record in Manchester Research Explorer

\section{Citation for published version (APA):}

Wang, C., \& Ding, Z. (2016). H Consensus Control of Multi-agent Systems with Input Delay and Directed

Topology. I E T Control Theory and Applications, 10(6), 617-624. https://doi.org/10.1049/iet-cta.2015.0945

\section{Published in:}

I E T Control Theory and Applications

\section{Citing this paper}

Please note that where the full-text provided on Manchester Research Explorer is the Author Accepted Manuscript or Proof version this may differ from the final Published version. If citing, it is advised that you check and use the publisher's definitive version.

\section{General rights}

Copyright and moral rights for the publications made accessible in the Research Explorer are retained by the authors and/or other copyright owners and it is a condition of accessing publications that users recognise and abide by the legal requirements associated with these rights.

\section{Takedown policy}

If you believe that this document breaches copyright please refer to the University of Manchester's Takedown Procedures [http://man.ac.uk/04Y6Bo] or contact uml.scholarlycommunications@manchester.ac.uk providing relevant details, so we can investigate your claim.

\section{OPEN ACCESS}




\title{
$H_{\infty}$ Consensus Control of Multi-agent Systems with Input Delay and Directed Topology
}

\author{
Chunyan Wang, Zhengtao Ding
}

\begin{abstract}
In this study, the consensus problem for linear multi-agent systems with general directed graph in the presence of constant input delay and external disturbances is addressed. To deal with input delay, a truncated prediction of the agent state over the delay period is approximated by the finite dimensional term of the classical state predictor. The truncated predictor feedback method is used for the consensus protocol design. By exploring certain features of the Laplacian matrix, the $H_{\infty}$ consensus analysis is put in the framework of Lyapunov analysis. The integral terms that remain in the transformed systems are carefully analyzed by using Krasovskii functional. Sufficient conditions are derived for the multi-agent systems to guarantee the $H_{\infty}$ consensus in the time domain. The feedback gain is then designed by solving these conditions with an iterative LMI procedure. A simulation study is carried out to validate the proposed control design.
\end{abstract}

\section{Index Terms}

$H_{\infty}$ consensus, multi-agent systems, truncated prediction, input delay, directed topology.

\section{INTRODUCTION}

With the recent advances in measurements and communication, cooperative control has received considerable attention in recent years, as evidenced by some recent publications [1]-[4]. In many applications, the control problems can be formulated as consensus problem for multi-agent systems with all the agents being coordinated to achieve a common control task [5].

Consensus problems have attracted significant interests from the control theory community and has been intensively studied in the literature. One significant contribution in consensus

C. Wang and Z. Ding are with School of Electrical and Electronic Engineering, University of Manchester, Sackville Street Building, Manchester M13 9PL, UK (email: chunyan.wang-2@postgrad.manchester.ac.uk; zhengtao.ding@manchester.ac.uk). 
control was due to the application of graph theory, particularly, the Laplacian matrix of the network connection, to the control design of the multi-agent systems [6]. Many early results on consensus problems are based on simple agent dynamics such as single integrators or secondorder dynamics [6]-[9]. The results were extended to high-order linear multi-agent systems in [10]-[13] and some for nonlinear systems [14]-[17].

The practical physical systems often suffer from external disturbances. The consensus problems of multi-agent systems with performance requirements have emerged as a challenge topic in recent years. The robust $H_{\infty}$ consensus problems were investigated for multi-agent systems with single integrators and second-order dynamics in [18], [19]. The $H_{\infty}$ consensus problems for general linear dynamics with undirected graphs were studied in [20], [21]. The results obtained in [20] were extended to directed graph in [22]. The $H_{\infty}$ consensus problems for switching directed topologies were investigated in [23], [24]. The nonlinear $H_{\infty}$ consensus problem was studied in [25] with directed graph. Global $H_{\infty}$ pinning synchronization problem for a class of directed networks with aperiodic sampled-data communications was addressed in [26]. It is worth noting that the directed graphs in [22]-[25] are restricted to be balanced or strongly connected. The main problem is that the Laplacian matrix associated with a directed graph is generally not positive semi-definite [27]. The decomposition method developed in [20] for the undirected systems cannot be applied to the directed one due to this unfavorable feature. Until now, it is still an active research area to achieve $H_{\infty}$ consensus control in general directed multi-agent systems.

Due to the time taken for the communications between the agents, delays are inevitable in the consensus control. In particular, the consensus time delay occurs in the control input when the protocols depend on the relative state information transmitted over the network. If not taken into consideration a priori, delays will degrade the performance of the closed-loop systems, and in the extreme situations, may even cause the loss of stability. Predictor feedback is a well-known approach to deal with the input delay. A wide variety of predictor-based methods [28]-[32] are effective for the control of input delayed systems. However, state prediction is based on the explicit solution of the state equation, which consists of the zero-input and the zero-state solutions. The zero-state solution involves the integral of the past control input and causes difficulty in control implementation [33]. An alternative method based on the prediction is to ignore the troublesome zero-state solution, and use the zero-input solution as the prediction. 
The resulting prediction is referred to as the truncated prediction [34]. The truncated prediction feedback method, which was originally developed for single linear systems [35]-[37], was later further developed for nonlinear systems [38], and for application in linear multi-agent systems [39], [40], where the open-loop dynamics of the agents is required to not exponentially unstable.

With this background, we consider the $H_{\infty}$ consensus control for high-order multi-agent systems with general directed graph and input delay. The contribution of this paper are two folds. Firstly, upon exploring the certain features of the Laplacian matrix in the real Jordan form, the $H_{\infty}$ consensus analysis is put in the framework of Lyapunov analysis for multi-agent systems connected by a general directed graph. Compared to the works in [22]-[25], the requirement for the communication graph in this paper is more general. The connection graph between the agents only needs a directed spanning tree, which is essential for consensus control, rather than the balanced or strongly connected conditions. Secondly, we consider the $H_{\infty}$ consensus control of multi-agent systems in the presence of input delay. This input delay may represent some delays in the network communication. By using the truncated prediction feedback method, the troublesome integral term is ignored, and only the prediction based on the exponential of the systems matrix is used for control design. Furthermore, rigorous analysis is carried out to ensure that the extra integral terms under the transformations, including the ones for external disturbances and input delay, are properly considered using Krasovskii functionals. Sufficient conditions are derived for the multi-agent systems to guarantee the $H_{\infty}$ consensus in the time domain. The conditions can be solved as LMIs (linear matrix inequalities) with a set of iterative scalar parameters. The iterative procedures developed in [43] for single linear system can be used here. A simulation study is carried out to demonstrate the results obtained in the paper.

The remainder of this paper is organized as follows. Section II presents some notations and the problem formulation. A few preliminary results for the consensus analysis are given in Section III. Section IV presents the main results on the $H_{\infty}$ consensus control design. Simulation results are given in Section V. Section VI concludes the paper.

\section{Problem Statement}

Consider a group of $N$ agents, each represented by a linear dynamic subject to input delay and external disturbance,

$$
\dot{x}_{i}(t)=A x_{i}(t)+B u_{i}(t-h)+D \omega_{i},
$$


where for agent $i, i=1,2, \ldots, N, x_{i} \in \mathbb{R}^{n}$ is the state vector, $u_{i} \in \mathbb{R}^{m \times n}$ is the control input vector, $A \in \mathbb{R}^{n \times n}, B \in \mathbb{R}^{n \times m}$ and $D \in \mathbb{R}^{n \times m}$ are constant matrices with $(A, B)$ being controllable, $h>0$ is input delay, and $\omega_{i} \in \mathcal{L}_{2}^{m}[0, \infty)$ is the external disturbance.

The communications among the agents are described by a directed graph $\mathcal{G}(\mathcal{V}, \mathcal{E})$, where $\mathcal{V}$ is a set of vertices and $\mathcal{E}$ is a set of edges. A vertex represents an agent, and each edge represents a connection. Associated with the graph is its adjacency matrix $Q$, where element $q_{i j}$ denotes the connection between two agents. More specifically, if a connection exists from agent $j$ to agent $i, q_{i j}=1$; otherwise $q_{i j}=0$. The Laplacian matrix $L$ is defined by $l_{i i}=\sum_{j=1}^{N} q_{i j}$ and $l_{i j}=-q_{i j}$ when $i \neq j$. For a directed graph, the Laplacian matrix $L$ has the following properties.

Lemma 1 ([8], [27]): The Laplacian matrix $L$ of a directed graph $\mathcal{G}$ has at least one zero eigenvalue with 1 as a corresponding right eigenvector and all nonzero eigenvalues have positive real parts. Furthermore, zero is a simple eigenvalue of $L$ if and only if $\mathcal{G}$ has a directed spanning tree. In addition, there exists a nonnegative left eigenvector $r$ of $L$ associated with the zero eigenvalue, satisfying $r^{T} L=0$ and $r^{T} \mathbf{1}=1$. Moreover, $r$ is unique if $\mathcal{G}$ has a directed spanning tree.

The objective of this paper is to design a control algorithm for each agent such that the multiagent systems (1) achieve consensus and meanwhile maintain a desirable disturbance rejection performance. In view of this, we introduce a state transformation

$$
\xi_{i}=x_{i}-\sum_{j=1}^{N} r_{j} x_{j},
$$

where $i=1,2, \ldots, N, \xi_{i} \in \mathbb{R}^{n}, r_{j}$ denotes the $j$ th element of $r$. Based on the new variable $\xi_{i}$, we define the performance variable as $e_{i}(t)=C \xi_{i}(t)$, where $e_{i} \in \mathbb{R}^{m}, C \in \mathbb{R}^{m \times n}$ is a constant matrix. Let $e(t)=\left[e_{1}^{T}, e_{2}^{T}, \cdots, e_{N}^{T}\right]^{T}, \omega=\left[\omega_{1}^{T}, \omega_{2}^{T}, \cdots, \omega_{N}^{T}\right]^{T}$. The $H_{\infty}$ consensus control problem can be defined as below.

Definition 1: Given a positive scalar $\gamma$, the $H_{\infty}$ consensus is achieved if the two requirements listed below are satisfied::

1. The multi-agent systems (1) with $\omega_{i} \equiv 0$ can reach consensus. That is, under these control algorithms, the following hold for all initial conditions,

$$
\lim _{t \rightarrow \infty}\left(x_{i}(t)-x_{j}(t)\right)=0, \quad \forall i \neq j .
$$


2. Under the zero-initial condition, the performance variable $e(t)$ satisfies

$$
J=\int_{0}^{\infty}\left[e^{T}(t) e(t)-\gamma^{2} \omega^{T}(t) \omega(t)\right] \mathrm{d} \tau<0 .
$$

Assumption 1: Zero is a simple eigenvalue of the Laplacian matrix $L$.

Remark 1: This assumption implies that the directed graph contains a spanning tree, which is essential for consensus control. If zero is not a simple eigenvalue of $L$, the agents cannot reach consensus asymptotically as there exist at least two separate subgroups or at least two agents in the group who do not receive any information [8].

Remark 2: The left eigenvector $r$ of the Laplacian matrix $L$ with the zero eigenvalue is crucial for the consensus design with directed graph. Feasible methods are given in [8], [16] to calculate this vector. In addition, the elements of $r$ could be zero. This suggests that the methods developed in [20]-[25] may not be suitable for the $H_{\infty}$ consensus analysis here.

\section{PRELiminary Results}

In this section, a few preliminary results are presented. We first recall the truncated predictor feedback method [34], [35]. Consider an input-delayed system

$$
\dot{x}(t)=A x(t)+B u(t-h) .
$$

From the system dynamics, we have

$$
x(t)=e^{A h} x(t-h)+\int_{t-h}^{t} e^{A(t-\tau)} B u(\tau-h) \mathrm{d} \tau .
$$

The first term, $e^{A h} x(t-h)$, is a truncated predictor of the state $x(t)$ based on $x(t-h)$. We take the control input as

$$
u(t)=K e^{A h} x(t)
$$

where $K \in \mathbb{R}^{m \times n}$ is a control gain matrix. The resultant closed-loop dynamics are given by

$$
\dot{x}(t)=(A+B K) x(t)-B K d(t),
$$

where

$$
d(t)=\int_{t-h}^{t} e^{A(t-\tau)} B u(\tau-h) \mathrm{d} \tau .
$$

In the truncated predictor feedback method, the troublesome integral term is ignored, and only the prediction based on the exponential of the systems matrix is used for control design. 
We also need the following results.

Lemma 2 ([41]): For a Laplacian matrix that satisfies Assumption 1, a similarity transformation $T$, with its first column $T_{(1)}=\mathbf{1}$ and the first row of $T^{-1}, T_{(1)}^{-1}=r^{T}$, can be constructed such that

$$
T^{-1} L T=\bar{J}
$$

with $\bar{J}$ being a block diagonal matrix in the real Jordan form

$$
\bar{J}=\left[\begin{array}{ccccccc}
0 & & & & & & \\
& \bar{J}_{1} & & & & & \\
& & \ddots & & & & \\
& & \bar{J}_{p} & & & \\
& & & \bar{J}_{p+1} & & \\
& & & & \ddots & \\
& & & & & \bar{J}_{q}
\end{array}\right],
$$

where $\bar{J}_{k} \in \mathbb{R}^{n_{k}}, k=1,2, \ldots, p$, are the Jordan blocks for real eigenvalues $\lambda_{k}>0$ with the multiplicity $n_{k}$ in the form

$$
\bar{J}_{k}=\left[\begin{array}{ccccc}
\lambda_{k} & 1 & & & \\
& \lambda_{k} & 1 & & \\
& & \ddots & \ddots & \\
& & & \lambda_{k} & 1 \\
& & & & \lambda_{k}
\end{array}\right]
$$

and $\bar{J}_{k} \in \mathbb{R}^{2 n_{k}}, k=p+1, p+2, \ldots, q$, are the Jordan blocks for conjugate eigenvalues $\alpha_{k} \pm j \beta_{k}$, $\alpha_{k}>0$ and $\beta_{k}>0$, with the multiplicity $n_{k}$ in the form

$$
\bar{J}_{k}=\left[\begin{array}{ccccc}
\nu\left(\alpha_{k}, \beta_{k}\right) & I_{2} & & & \\
& \nu\left(\alpha_{k}, \beta_{k}\right) & I_{2} & & \\
& & \ddots & \ddots & \\
& & & \nu\left(\alpha_{k}, \beta_{k}\right) & I_{2} \\
& & & & \nu\left(\alpha_{k}, \beta_{k}\right)
\end{array}\right]
$$

with $I_{2}$ being the identity matrix in $\mathbb{R}^{2 \times 2}$ and

$$
\nu\left(\alpha_{k}, \beta_{k}\right)=\left[\begin{array}{cc}
\alpha_{i} & \beta_{i} \\
-\beta_{i} & \alpha_{i}
\end{array}\right] \in \mathbb{R}^{2 \times 2} .
$$


Lemma 3 ([42]): For a positive definite matrix $P$, and a function $x:[a, b] \rightarrow \mathbb{R}^{n}$, with $a, b \in \mathbb{R}$ and $b>a$, the following inequality holds:

$$
\left(\int_{a}^{b} x^{T}(\tau) \mathrm{d} \tau\right) P\left(\int_{a}^{b} x(\tau) \mathrm{d} \tau\right) \leq(b-a) \int_{a}^{b} x^{T}(\tau) P x(\tau) \mathrm{d} \tau .
$$

Lemma 4 ([36], [38]): For a positive definite matrix $P$, the following identity holds

$$
e^{A^{T} t} P e^{A t}-e^{\lambda t} P=-e^{\lambda t} \int_{0}^{t} e^{-\lambda \tau} e^{A^{T} \tau} R e^{A \tau} \mathrm{d} \tau
$$

where

$$
R=-A^{T} P-P A+\lambda P .
$$

Furthermore, if $R$ is positive definite, $\forall t>0$,

$$
e^{A^{T} t} P e^{A t}<e^{\lambda t} P
$$

\section{Consensus Control}

For the multi-agent system (1), we have

$$
x_{i}=e^{A h} x_{i}(t-h)+\int_{t-h}^{t} e^{A(t-\tau)}\left(B u_{i}(\tau-h)+D \omega_{i}\right) \mathrm{d} \tau .
$$

The control input takes the structure

$$
u_{i}(t)=K e^{A h} \sum_{j=1}^{N} q_{i j}\left(x_{i}(t)-x_{j}(t)\right)=K e^{A h} \sum_{j=1}^{N} l_{i j} x_{j}(t),
$$

where $K \in \mathbb{R}^{m \times n}$ is a constant control gain to be designed later. Under control algorithm (9), the multi-agent system (1) can be written as

$$
\dot{x}_{i}=A x_{i}+B K \sum_{j=1}^{N} l_{i j} x_{j}+D \omega_{i}-B K \sum_{j=1}^{N} l_{i j} \int_{t-h}^{t} e^{A(t-\tau)}\left(B u_{j}(\tau-h)+D \omega_{j}\right) \mathrm{d} \tau .
$$

Let $x=\left[x_{1}^{T}, x_{2}^{T}, \cdots, x_{N}^{T}\right]^{T}, u=\left[u_{1}^{T}, u_{2}^{T}, \cdots, u_{N}^{T}\right]^{T}$. The closed-loop system is then described by

$$
\dot{x}=\left(I_{N} \otimes A+L \otimes B K\right) x+(L \otimes B K)\left(d_{1}+d_{2}\right)+\left(I_{N} \otimes D\right) \omega
$$

where $d_{1}=-\int_{t-h}^{t}\left(I_{N} \otimes e^{A(t-\tau)} B\right) u(\tau-h) \mathrm{d} \tau, d_{2}=-\int_{t-h}^{t}\left(I_{N} \otimes e^{A(t-\tau)} D\right) \omega(t) \mathrm{d} \tau$, and $\otimes$ denotes the Kronecker product of matrices.

From the state transformation (2), we have

$$
\xi=x-\left(\left(1 r^{T}\right) \otimes I_{n}\right) x=\left(U \otimes I_{n}\right) x
$$


where $\xi=\left[\xi_{1}^{T}, \xi_{2}^{T}, \cdots, \xi_{N}^{T}\right]^{T}, U=I_{N}-\mathbf{1} r^{T}$. Since $r^{T} \mathbf{1}=1$, it can be shown that $U \mathbf{1}=0$. Therefore the consensus of system (1) is achieved when $\lim _{t \rightarrow \infty} \xi(t)=0$, as $\xi=0$ implies that $x_{1}=x_{2}=\cdots=x_{N}$, due to the fact that the null space of $U$ is $\operatorname{span}\{\mathbf{1}\}$. The dynamics of $\xi$ can then be derived as

$$
\dot{\xi}=\left(I_{N} \otimes A+L \otimes B K\right) \xi+(L \otimes B K)\left(d_{1}+d_{2}\right)+(U \otimes D) \omega
$$

where we have used $r^{T} L=0$.

To explore the structure of $L$, we propose another state transformation

$$
\eta=\left(T^{-1} \otimes I_{n}\right) \xi
$$

with $\eta=\left[\eta_{1}^{T}, \eta_{2}^{T}, \cdots, \eta_{N}^{T}\right]^{T}$. Then we have

$$
\dot{\eta}=\left(I_{N} \otimes A+\bar{J} \otimes B K\right) \eta+\Delta_{1}(x)+\Delta_{2}(t)+\Omega(t),
$$

where

$$
\begin{aligned}
& \Delta_{1}=\left(T^{-1} L \otimes B K\right) d_{1}, \\
& \Delta_{2}=\left(T^{-1} L \otimes B K\right) d_{2}, \\
& \Omega=\left(T^{-1} U \otimes D\right) \omega(t) .
\end{aligned}
$$

From state transformations (11) and (13), we have:

$$
\eta_{1}=\left(r^{T} \otimes I_{n}\right) \xi=\left(\left(r^{T} U\right) \otimes I_{n}\right) x \equiv 0 .
$$

With the control law shown in (9), the control gain matrix $K$ is chosen as

$$
K=-B^{T} P
$$

where $P$ is a positive definite matrix to be designed. In the remainder of the paper, Lyapunovfunction-based analysis will be carried out to identify a condition for $P$ to ensure that the consensus problem is solved by using the control algorithm (15) with control gain $K$ in (9).

The consensus analysis will be carried out in terms of $\eta$. Let

$$
V_{i}=\eta_{i}^{T} P \eta_{i}
$$

for $i=2,3, \cdots, N$. Then, let

$$
V_{0}=\sum_{i=2}^{N} V_{i}
$$


For the convenience of presentation, we recall from [41] the following results on $V_{0}$.

Lemma 5: For multi-agent systems (1) with the transformed state $\eta, \dot{V}_{0}$ has following bounds specified in one of the following two cases:

1) If the eigenvalues of the Laplacian matrix $L$ are distinct, $\dot{V}_{0}$ satisfies

$$
\begin{aligned}
\dot{V}_{0} \leq & \sum_{i=2}^{N} \eta_{i}^{T}\left(A^{T} P+P A-2 \alpha P B B^{T} P+\left(\kappa_{1}+\kappa_{2}\right) P P\right) \eta_{i} \\
& +\frac{1}{\kappa_{1}}\left\|\Delta_{1}\right\|^{2}+\frac{1}{\kappa_{2}}\left\|\Delta_{2}\right\|^{2}+2 \eta^{T}\left(I_{N} \otimes P\right) \Omega
\end{aligned}
$$

where $\kappa_{1}$ and $\kappa_{2}$ are any positive real numbers, and

$$
\alpha=\min \left\{\lambda_{1}, \lambda_{2}, \ldots, \lambda_{p}, \alpha_{p+1}, \alpha_{p+2}, \ldots, \alpha_{q}\right\}
$$

2) If the Laplacian matrix $L$ has multiple eigenvalues, $\dot{V}_{0}$ satisfies

$$
\begin{aligned}
\dot{V}_{0} \leq & \sum_{i=2}^{N} \eta_{i}^{T}\left(A^{T} P+P A-2(\alpha-1) P B B^{T} P+\left(\kappa_{1}+\kappa_{2}\right) P P\right) \eta_{i} \\
& +\frac{1}{\kappa_{1}}\left\|\Delta_{1}\right\|^{2}+\frac{1}{\kappa_{2}}\left\|\Delta_{2}\right\|^{2}+2 \eta^{T}\left(I_{N} \otimes P\right) \Omega,
\end{aligned}
$$

where $\kappa_{1}$ and $\kappa_{2}$ are any positive real numbers.

The following lemmas give the bounds of $\left\|\Delta_{1}\right\|^{2}$ and $\left\|\Delta_{2}\right\|^{2}$.

Lemma 6: For the term $\Delta_{1}(x)$ shown in the transformed system dynamics (14), a bound can be established as

$$
\left\|\Delta_{1}\right\|^{2} \leq \rho_{1} \int_{t-h}^{t} \eta^{T}(\tau-h) \eta(\tau-h) \mathrm{d} \tau
$$

where

$$
\rho_{1}=4 h a_{1}^{4} e^{2 \lambda h} \lambda_{\sigma}^{2}\left(T^{-1}\right)\|L\|_{F}^{2}\|Q\|_{F}^{2}\|T\|_{F}^{2},
$$

with $a_{1}$ and $\lambda$ being positive numbers such that

$$
\begin{gathered}
a_{1}^{2} I \geq P B B^{T} B B^{T} P \\
\lambda I>A^{T}+A
\end{gathered}
$$

and $\lambda_{\sigma}(\cdot)$ and $\|\cdot\|_{F}$ being the maximum singular value and the Frobenius norm of a matrix, respectively. 
Proof: By the definition of $\Delta_{1}(x)$ in (14), we have

$$
\begin{aligned}
\left\|\Delta_{1}\right\| & =\left\|\left(T^{-1} \otimes I_{n}\right)(L \otimes B K) d_{1}\right\| \\
& \leq \lambda_{\sigma}\left(T^{-1}\right)\|\mu\|,
\end{aligned}
$$

where $\mu=(L \otimes B K) d_{1}$.

Let $\mu=\left[\mu_{1}^{T}, \mu_{2}^{T}, \ldots, \mu_{N}^{T}\right]^{T}$. Then from (10) and (15), we have

$$
\mu_{i}=B B^{T} P \sum_{j=1}^{N} l_{i j} \int_{t-h}^{t} e^{A(t-\tau)} B B^{T} P e^{A h} \sum_{k=1}^{N} q_{j k}\left(x_{k}(\tau-h)-x_{j}(\tau-h)\right) \mathrm{d} \tau .
$$

From $\eta=\left(T^{-1} \otimes I_{n}\right) \xi$, we obtain $\xi=\left(T \otimes I_{n}\right) \eta$. And from the state transformation (11), we have

$$
\begin{aligned}
x_{k}(t)-x_{j}(t) & =\xi_{k}(t)-\xi_{j}(t) \\
& =\left(\left(T_{k}-T_{j}\right) \otimes I_{n}\right) \eta(t) \\
& =\sum_{l=1}^{N}\left(T_{k l}-T_{j l}\right) \eta_{l}(t),
\end{aligned}
$$

where $T_{k}$ denotes the $k$ th row of $T$.

We define

$$
\sigma_{l}=B B^{T} P \int_{t-h}^{t} \mathrm{e}^{A(t-\tau)} B B^{T} P e^{A h} \eta_{l}(\tau-h) \mathrm{d} \tau .
$$

Then, from (23) and (24), we can obtain that

$$
\mu_{i}=\sum_{j=1}^{N} l_{i j} \sum_{k=1}^{N} q_{j k} \sum_{l=1}^{N}\left(T_{k l}-T_{j l}\right) \sigma_{l}
$$

For the notational convenience, let $\sigma=\left[\sigma_{1}^{T}, \sigma_{2}^{T}, \ldots, \sigma_{N}^{T}\right]^{T}$. It then follows that

$$
\begin{aligned}
\left\|\mu_{i}\right\| & \leq \sum_{j=1}^{N}\left|l_{i j}\right| \sum_{k=1}^{N}\left|q_{j k}\right|\left\|T_{k}\right\|\|\sigma\|+\sum_{k=1}^{N} \sum_{j=1}^{N}\left|l_{i j}\right|\left|q_{j k}\right|\left\|T_{j}\right\|\|\sigma\| \\
& \leq \sum_{j=1}^{N}\left|l_{i j}\right|\left\|q_{j}\right\|\|T\|_{F}\|\sigma\|+\sum_{k=1}^{N} \sum_{j=1}^{N}\left|l_{i j}\right|\left\|q_{k}\right\|\|T\|_{F}\|\sigma\| \\
& \leq 2\left\|l_{i}\right\|\|Q\|_{F}\|T\|_{F}\|\sigma\|
\end{aligned}
$$

where $l_{i}$ denotes the $i$ th row of $L$. Therefore we have

$$
\|\mu\|^{2}=\sum_{i=1}^{N}\left\|\mu_{i}\right\|^{2} \leq 4\|L\|_{F}^{2}\|Q\|_{F}^{2}\|T\|_{F}^{2}\|\sigma\|^{2}
$$


where we have used $\sum_{i=1}^{N}\left\|l_{i}\right\|^{2}=\left(\|L\|_{F}\right)^{2}$.

Next we need to deal with $\|\sigma\|^{2}$. By Lemma 3, we have

$$
\begin{aligned}
\left\|\sigma_{i}\right\|^{2} & \leq h \int_{t-h}^{t} \eta_{i}^{T}(\tau-h) e^{A^{T} h} P B B^{T} e^{A^{T}(t-\tau)} P B B^{T} B B^{T} P e^{A(t-\tau)} B B^{T} P e^{A h} \eta_{i}(\tau-h) \mathrm{d} \tau \\
& \leq h a_{1}^{2} \int_{t-h}^{t} \eta_{i}^{T}(\tau-h) e^{A^{T} h} P B B^{T} e^{A^{T}(t-\tau)} e^{A(t-\tau)} B B^{T} P e^{A h} \eta_{i}(\tau-h) \mathrm{d} \tau,
\end{aligned}
$$

where $a_{1}$ is a positive real number such that

$$
a_{1}^{2} I \geq P B B^{T} B B^{T} P .
$$

In view of Lemma 4 with $P=I$, provided that

$$
R=-A^{T}-A+\lambda I>0,
$$

we have

$$
e^{A^{T} t} e^{A t}<e^{\lambda t} I,
$$

and

$$
\begin{aligned}
\left\|\sigma_{i}\right\|^{2} & \leq h a_{1}^{2} \int_{t-h}^{t} e^{\lambda(t-\tau)} \eta_{i}^{T}(\tau-h) e^{A^{T} h} P B B^{T} B B^{T} P e^{A h} \eta_{i}(\tau-h) \mathrm{d} \tau \\
& \leq h a_{1}^{4} e^{2 \lambda h} \int_{t-h}^{t} \eta_{i}^{T}(\tau-h) \eta_{i}(\tau-h) \mathrm{d} \tau .
\end{aligned}
$$

Then, $\|\sigma\|^{2}$ can be bounded as

$$
\begin{aligned}
\|\sigma\|^{2} & =\sum_{i=1}^{N}\left\|\sigma_{i}\right\|^{2} \\
& \leq h a_{1}^{4} e^{2 \lambda h} \int_{t-h}^{t} \eta^{T}(\tau-h) \eta(\tau-h) \mathrm{d} \tau .
\end{aligned}
$$

Hence, together with (22), (27) and (28), we get

$$
\left\|\Delta_{1}\right\|^{2} \leq \rho_{1} \int_{t-h}^{t} \eta^{T}(\tau-h) \eta(\tau-h) \mathrm{d} \tau .
$$

This completes the proof.

Lemma 7: For the term $\Delta_{2}(t)$ in the transformed system dynamics (14), a bound can be established as

$$
\left\|\Delta_{2}\right\|^{2} \leq \rho_{2} \int_{t-h}^{t} \omega^{T}(\tau) \omega(\tau) \mathrm{d} \tau
$$


where

$$
\rho_{2}=h a_{1}^{2} a_{2} e^{\lambda h} \lambda_{\sigma}^{2}\left(T^{-1}\right)\|L\|_{F}^{2},
$$

with $a_{1}$ and $\lambda$ being as defined in (20) and (21), and $a_{2}$ is a positive real number such that

$$
a_{2} I \geq D^{T} D \text {. }
$$

Proof: In a way similar to Lemma 3, we have

$$
\begin{aligned}
\left\|\Delta_{2}\right\| & =\left\|\left(T^{-1} \otimes I_{n}\right)(L \otimes B K) d_{2}\right\| \\
& \leq \lambda_{\sigma}\left(T^{-1}\right)\|z\|,
\end{aligned}
$$

where $z=(L \otimes B K) d_{2}$. Let $z=\left[z_{1}^{T}, z_{2}^{T}, \ldots, z_{N}^{T}\right]^{T}$. Then from (10) and (15), we have

$$
z_{i}=\sum_{j=1}^{N} l_{i j} B B^{T} P \int_{t-h}^{t} e^{A(t-\tau)} D \omega_{j} \mathrm{~d} \tau
$$

It follows that

$$
\left\|z_{i}\right\|^{2}=\sum_{j=1}^{N} l_{i j}^{2} \int_{t-h}^{t} \omega_{j}^{T} D^{T} e^{A^{T}(t-\tau)} \mathrm{d} \tau P B B^{T} B B^{T} P \int_{t-h}^{t} e^{A(t-\tau)} D \omega_{j} \mathrm{~d} \tau .
$$

With Lemma 3 and the condition (20), we have

$$
\left\|z_{i}\right\|^{2} \leq h a_{1}^{2} \sum_{j=1}^{N} l_{i j}^{2} \int_{t-h}^{t} \omega_{j}^{T} D^{T} e^{A^{T}(t-\tau)} e^{A(t-\tau)} D \omega_{j} \mathrm{~d} \tau .
$$

In view of Lemma 4, with the conditions (21) and (30), we have

$$
\left\|z_{i}\right\|^{2} \leq h a_{1}^{2} a_{2} e^{\lambda h} \int_{t-h}^{t} \sum_{j=1}^{N} l_{i j}^{2}\left\|\omega_{j}\right\|^{2} \mathrm{~d} \tau .
$$

Consequently,

$$
\begin{aligned}
\|z\|^{2} & \leq h a_{1}^{2} a_{2} e^{\lambda h} \int_{t-h}^{t} \sum_{i=1}^{N} \sum_{j=1}^{N} l_{i j}^{2}\left\|\omega_{j}\right\|^{2} \mathrm{~d} \tau \\
& \leq h a_{1}^{2} a_{2} e^{\lambda h}\|L\|_{F}^{2} \int_{t-h}^{t} \omega^{T}(\tau) \omega(\tau) \mathrm{d} \tau .
\end{aligned}
$$

Putting (31) and (32) together, we have

$$
\left\|\Delta_{2}\right\|^{2} \leq \rho_{2} \int_{t-h}^{t} \omega^{T}(\tau) \omega(\tau) \mathrm{d} \tau
$$

This completes the proof. 
For the first integral term shown in (19), we consider the following Krasovskii functional

$$
W_{1}=e^{h} \int_{t-h}^{t} \eta^{T}(\tau) \eta(\tau) \mathrm{d} \tau+e^{h} \int_{t-h}^{t} e^{\tau-t} \eta^{T}(\tau-h) \eta(\tau-h) \mathrm{d} \tau .
$$

A direct evaluation gives that

$$
\begin{aligned}
\dot{W}_{1} & =-e^{h} \int_{t-h}^{t} e^{\tau-t} \eta^{T}(\tau-h) \eta(\tau-h) \mathrm{d} \tau-\eta^{T}(t-2 h) \eta(t-2 h)+e^{h} \eta^{T}(t) \eta(t) \\
& \leq-\int_{t-h}^{t} \eta^{T}(\tau-h) \eta(\tau-h) \mathrm{d} \tau+e^{h} \eta(t)^{T} \eta(t) .
\end{aligned}
$$

For the second integral term shown in (29), we consider the following Krasovskii functional

$$
W_{2}=e^{h} \int_{t-h}^{t} e^{\tau-t} \omega^{T}(\tau) \omega(\tau) \mathrm{d} \tau
$$

A direct evaluation gives that

$$
\begin{aligned}
\dot{W}_{2} & =-e^{h} \int_{t-h}^{t} e^{\tau-t} \omega^{T}(\tau) \omega(\tau) \mathrm{d} \tau+e^{h} \omega^{T}(\tau) \omega(\tau)-\omega^{T}(t-h) \omega(t-h) \\
& \leq-\int_{t-h}^{t} \omega^{T}(\tau) \omega(\tau) \mathrm{d} \tau+e^{h} \omega^{T}(\tau) \omega(\tau) .
\end{aligned}
$$

Let

$$
V=V_{0}+\frac{\rho_{1}}{\kappa_{1}} W_{1}+\frac{\rho_{2}}{\kappa_{2}} W_{2}
$$

From (17), (18), (33) and (34), we obtain that

$$
\dot{V} \leq \eta^{T}(t)\left[I_{N} \otimes\left(H+\frac{\rho_{1}}{\kappa_{1}} e^{h} I_{n}\right)\right] \eta(t)+\frac{\rho_{2}}{\kappa_{2}} e^{h} \omega^{T}(t) \omega(t)+2 \eta^{T}\left(I_{N} \otimes P\right) \Omega,
$$

where

$$
H:=A^{T} P+P A-2 \alpha P B B^{T} P+\left(\kappa_{1}+\kappa_{2}\right) P P,
$$

for Case 1), and

$$
H:=A^{T} P+P A-2(\alpha-1) P B B^{T} P+\left(\kappa_{1}+\kappa_{2}\right) P P
$$

for Case 2).

The above expressions can be used for the $H_{\infty}$ consensus analysis. The following theorem summarizes the results.

Theorem 1: For an input-delayed multi-agent system (1) with the associated Laplacian matrix that satisfies Assumption 1, the $H_{\infty}$ consensus control problem can be solved by the control algorithm (9) with the control gain $K=-B^{T} P$ specified in one of the following two cases: 
1) If the eigenvalues of the Laplacian matrix $L$ are distinct, the consensus is achieved if the following conditions are satisfied for $W=P^{-1}$ and $a_{1}>0, \lambda \geq 0$,

$$
\begin{aligned}
& \left(A-\frac{1}{2} \lambda I_{n}\right)^{T}+\left(A-\frac{1}{2} \lambda I_{n}\right)<0, \\
& a_{1} W \geq B B^{T}, \\
& {\left[\begin{array}{ccc}
\Gamma_{1} & W & D \\
W & -\left(\frac{\rho_{1} e^{h}}{\kappa_{1}}+a_{3}\right)^{-1} I_{n} & 0 \\
D^{T} & 0 & -\left(\gamma^{2}-\frac{\rho_{2}}{\kappa_{2}} e^{h}\right) a_{4}^{-1}
\end{array}\right]<0,}
\end{aligned}
$$

where $\Gamma_{1}=W A^{T}+A W-2 \alpha B B^{T}+\left(\kappa_{1}+\kappa_{2}\right) I_{n}, \kappa_{1}>0, \kappa_{2}>\frac{\rho_{2}}{\gamma^{2}} e^{h}, a_{3} \geq \lambda_{\max }\left(T^{T} T \otimes C^{T} C\right)$ and $a_{4} \geq \lambda_{\max }\left(T^{-1} U U^{T}\left(T^{-1}\right)^{T}\right)$.

2) If the Laplacian matrix $L$ has multiple eigenvalues, the consensus is achieved if the conditions (37), (38) and the following condition are satisfied for $W=P^{-1}, a_{1}>0, \lambda \geq 0$,

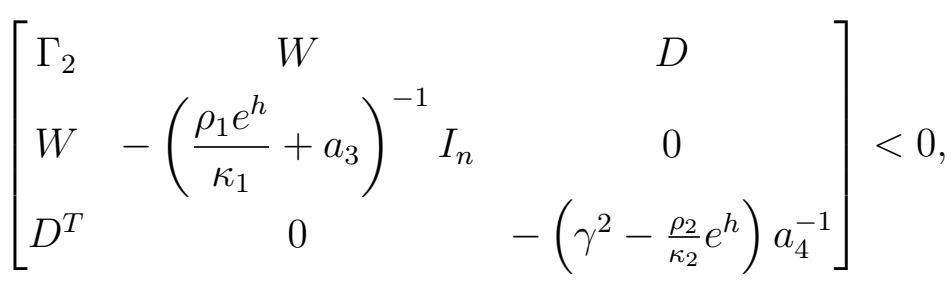

where $\Gamma_{2}=W A^{T}+A W-2(\alpha-1) B B^{T}+\left(\kappa_{1}+\kappa_{2}\right) I_{n}$.

Proof: From (13), we obtain that

$$
e(t)=\left(I_{N} \otimes C\right) \xi=\left(I_{N} \otimes C\right)\left(T \otimes I_{n}\right) \eta(t) .
$$

It follows that

$$
e^{T}(t) e(t)=\eta^{T}(t)\left(T^{T} T \otimes C^{T} C\right) \eta(t) \leq a_{3} \eta^{T}(t) \eta(t) .
$$

Under the zero-initial condition, $x(0)=0$. It is clear that $V(0)=0$. Next, for any non-zero $\omega$, 
we have

$$
\begin{aligned}
J= & \int_{0}^{\infty}\left[e^{T}(t) e(t)-\gamma^{2} \omega^{T}(t) \omega(t)+\dot{V}\right] \mathrm{d} \tau-V(\infty)+V(0) \\
\leq & \int_{0}^{\infty} \eta^{T}\left[I_{N} \otimes\left(H+\frac{\rho_{1}}{\kappa_{1}} e^{h} I_{n}+a_{3} I_{n}\right)\right] \eta \mathrm{d} \tau \\
& +\int_{0}^{\infty}\left(\frac{\rho_{2}}{\kappa_{2}} e^{h}-\gamma^{2}\right) \omega^{T}(t) \omega(t)+2 \eta^{T}\left(I_{N} \otimes P\right) \Omega \mathrm{d} \tau \\
= & \int_{0}^{\infty}\left[\begin{array}{c}
\eta \\
\omega
\end{array}\right]^{T} \Theta\left[\begin{array}{c}
\eta \\
\omega
\end{array}\right] \mathrm{d} \tau,
\end{aligned}
$$

where

$$
\Theta=\left[\begin{array}{cc}
I_{N} \otimes\left(H+\frac{\rho_{1}}{\kappa_{1}} e^{h} I_{n}+a_{3} I_{n}\right) & T^{-1} U \otimes P D \\
\left(T^{-1} U\right)^{T} \otimes D^{T} P & \left(\frac{\rho_{2}}{\kappa_{2}} e^{h}-\gamma^{2}\right) I
\end{array}\right] .
$$

Thus, $J<0$ if $\Theta<0$. By Schur complement lemma, we know that $\Theta<0$ if the following inequality hold

$$
H+\frac{\rho_{1}}{\kappa_{1}} e^{h} I_{n}+a_{3} I_{n}+a_{4}\left(\gamma^{2}-\frac{\rho_{2}}{\kappa_{2}} e^{h}\right)^{-1} P D D^{T} P<0 .
$$

From $W=P^{-1}$, and condition (36), it is obtained that (43) is equivalent to

$$
\begin{aligned}
W A^{T} & +A W-2 \alpha B B^{T}+\left(\kappa_{1}+\kappa_{2}\right) I_{n} \\
& +\left(\frac{\rho_{1}}{\kappa_{1}} e^{h}+a_{3}\right) W W+a_{4}\left(\gamma^{2}-\frac{\rho_{2}}{\kappa_{2}} e^{h}\right)^{-1} D D^{T}<0,
\end{aligned}
$$

for Case 1), and

$$
\begin{aligned}
W A^{T} & +A W-2(\alpha-1) B B^{T}+\left(\kappa_{1}+\kappa_{2}\right) I_{n} \\
& +\left(\frac{\rho_{1}}{\kappa_{1}} e^{h}+a_{3}\right) W W+a_{4}\left(\gamma^{2}-\frac{\rho_{2}}{\kappa_{2}} e^{h}\right)^{-1} D D^{T}<0,
\end{aligned}
$$

for Case 2).

By Schur complement lemma, we know that the conditions (44) and (45) are equivalent to the conditions specified in (39) and (40). Considering conditions (37)-(40), we can obtain that $J<0$. Therefore the $H_{\infty}$ consensus problem is solved.

The consensus analysis for multi-agent systems with directed graph can clearly be applied to the systems with undirected graphs. Indeed, it can be treated as a special situation of the Case 1. A corollary is given for this special case.. 


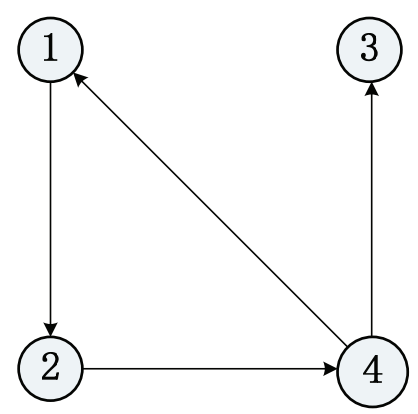

Fig. 1. Communication topology.

Corollary 1: For an input-delayed multi-agent system (1) with undirected graph, the $H_{\infty}$ consensus control problem can be solved by the control algorithm (9) with the control gain $K=-B^{T} P$ where $P$ is a positive definite matrix satisfying conditions (37)-(39).

Remark 3: Due to the nonlinear matrix inequalities, traditional LMI solvers cannot be used to find a solution for Theorem 1. Instead, the conditions shown in (37) to (40) can be checked by following the iterative methods developed in [43].

\section{Simulation}

In this section, A simulation study is carried out to demonstrate the effectiveness of the proposed control design. Consider a connection of four agents as shown in Figure 1. The dynamics of each agent is described by (1), with

$$
A=\left[\begin{array}{cc}
0 & -0.1 \\
0.1 & 0.1
\end{array}\right], B=\left[\begin{array}{cc}
0.5 & 0 \\
0 & 0.5
\end{array}\right], C=\left[\begin{array}{c}
0.1 \\
0
\end{array}\right]^{T}, D=\left[\begin{array}{cc}
0.1 & 0 \\
0 & 0.1
\end{array}\right]
$$

Note that $A$ has two positive eigenvalues. The external disturbances $\omega=[2 w, w,-2 w, 1.5 w]^{T}$, where $w(t)$ is a ten-period square wave starting at $t=0$ with the width 5 and height 1 . The input delay of the system is 0.1 seconds. The Laplacian matrix is given by

$$
L=\left[\begin{array}{cccc}
1 & 0 & 0 & -1 \\
-1 & 1 & 0 & 0 \\
0 & 0 & 1 & -1 \\
0 & -1 & 0 & 1
\end{array}\right]
$$




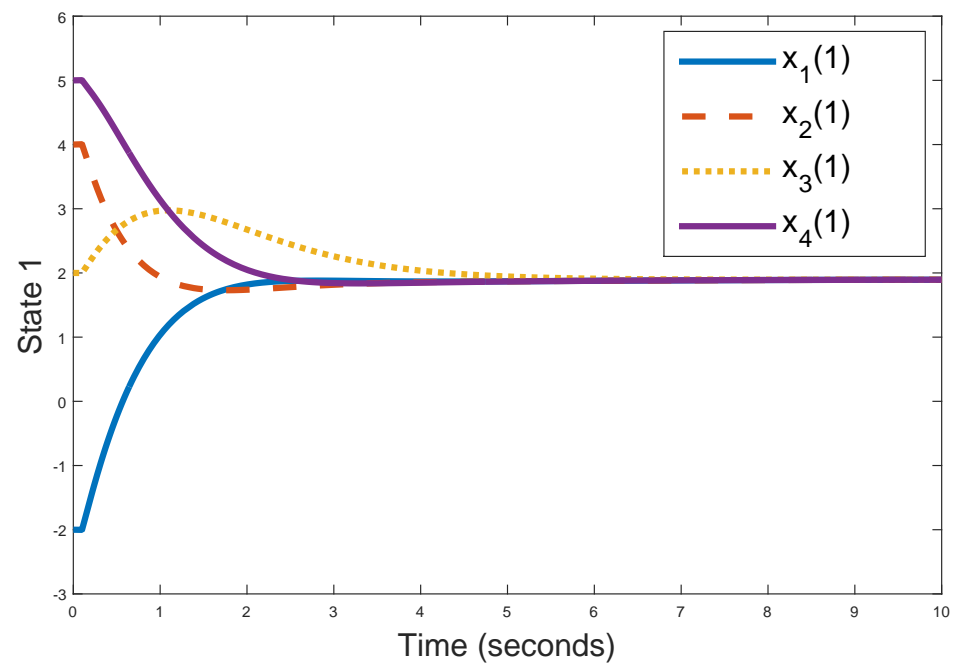

Fig. 2. The state 1 of agents with $h=0.1$.

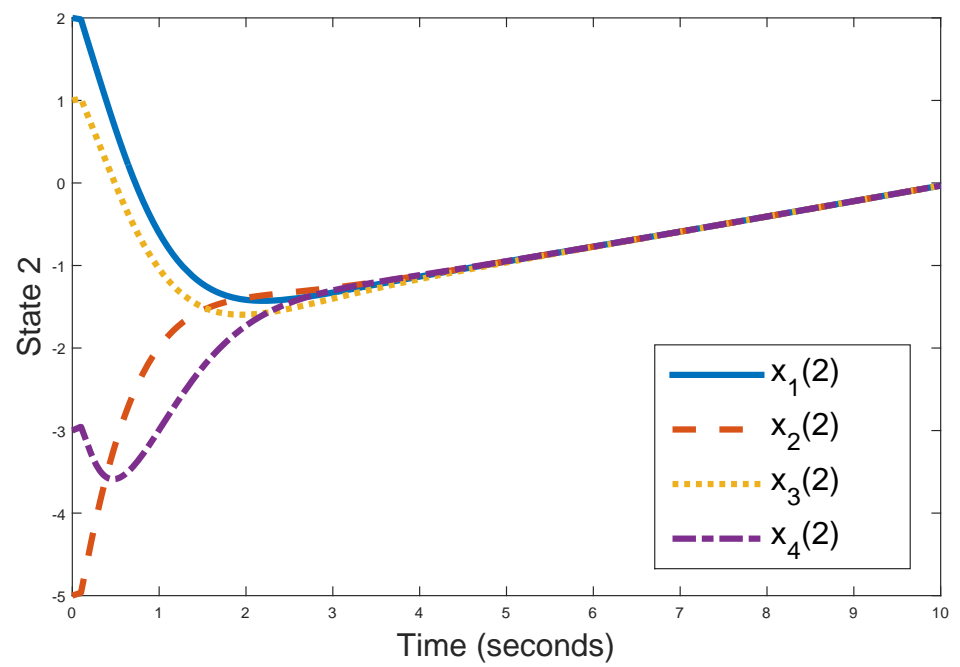

Fig. 3. The state 2 of agents with $h=0.1$.

The eigenvalues of $L$ are $\{0,1,3 / 2 \pm j \sqrt{3} / 2\}$. Therefore, Assumption 1 is satisfied. We obtain that $\alpha=1$ and $r^{T}=\left[\frac{1}{3}, \frac{1}{3}, 0, \frac{1}{3}\right]$. In this case, we choose the $H_{\infty}$ performance index

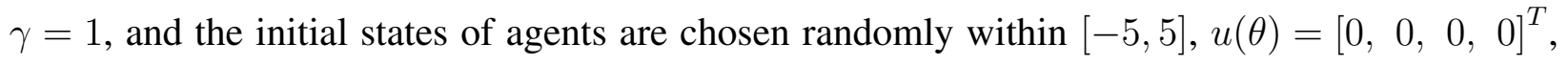
for $\theta \in[-h, 0]$. With the values of $\lambda=0.2, a_{1}=0.3$, and $\kappa_{1}=\kappa_{2}=0.1$, a feasible solution of 


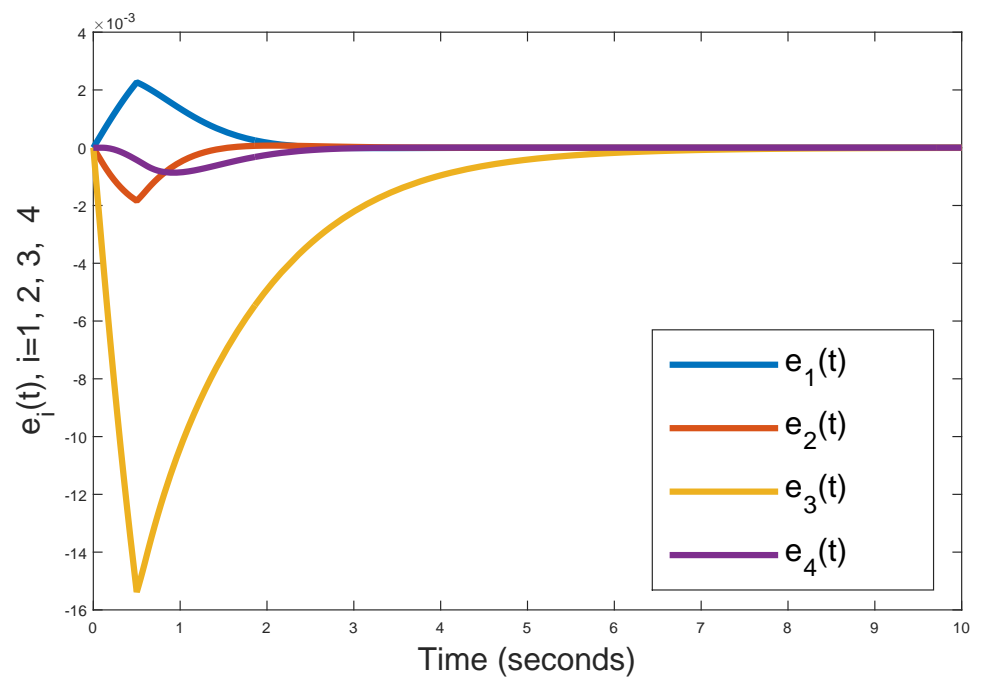

Fig. 4. The trajectories of performance variables.

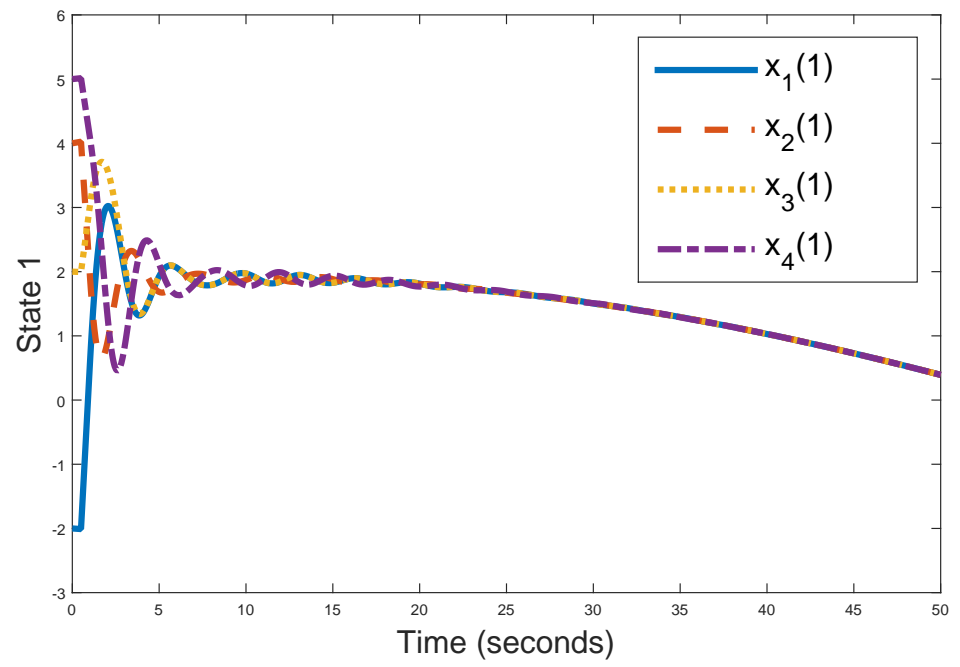

Fig. 5. The state 1 of agents with $h=0.5$.

the feedback gain $K$ is found to be

$$
K=\left[\begin{array}{ll}
-1.5349 & -0.1504 \\
-0.1504 & -1.5541
\end{array}\right] \text {. }
$$

Figures 2 and 3 show the simulation results for the state of each agent under the case $\omega=0$. Clearly the conditions specified in Theorem 1 are sufficient for the control gain to achieve con- 


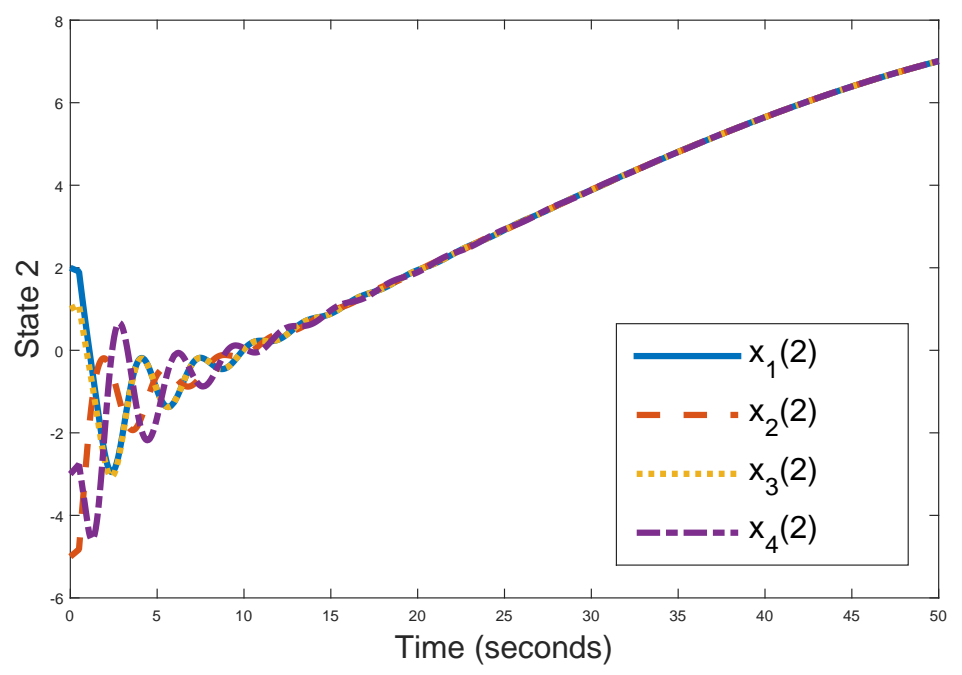

Fig. 6. The state 2 of agents with $h=0.5$.

sensus control. Figures 4 shows the trajectories of the performance variables $e_{i}(t), i=1, \cdots, 4$ under the zero-initial condition. In addition, with the same control gain, the consensus control is still achieved for the multi-agent system with a much larger delay $h=0.5$, as shown in Figures 5 and 6, which implies the conditions could be conservative in the control gain design for a given input delay. Further investigation to relax the conditions is a topic of future research.

\section{CONCLUSION}

In this paper, we have addressed the $H_{\infty}$ consensus problem for linear multi-agent systems with input delay and general directed graph. This input delay may represent some delays in the network communication. The truncated prediction feedback method is employed to deal with the input delay, and the integral terms that remain in the transformed systems are carefully analyzed by using Krasovskii functionals. By using the real Jordan form of the Laplacian matrix, sufficient conditions for the $H_{\infty}$ consensus are identified through Lyapunov analysis. The conditions can be solved by employing LMIs with a set of iterative parameters. The requirement for the communication graph in this paper is much relaxed than the conditions specified in [22]-[25], as the method presented in this paper only requires the connection graph to have a spanning tree. Future work will focus on $H_{\infty}$ consensus protocol design for multi-agent systems with time-varying input delay and Lipschitz nonlinearities. 


\section{REFERENCES}

[1] Zhang, H., Lewis, F., Das, A.: 'Optimal design for synchronization of cooperative systems: state feedback, observer and output feedback', IEEE Trans. Autom. Control, 2011, 56, (8), pp. 1948-1552

[2] Qu, Z.: 'Cooperative Control of Dynamical Systems: Applications to Autonomous Vehicles' (Springer-Verlag, London, 2009)

[3] Martin, S., Girard, A., Fazeli, A., Jadbabaie, A.: 'Multiagent flocking under general communication rule', IEEE Trans. Control Netw. Syst., 2014, 1, (2), pp. 155-166

[4] Dong, X., Shi, Z., Lu, G., Zhong, Y.: 'Time-varying formation control for high-order linear swarm systems with switching interaction topologies', IET Control Theory Appl., 2014, 8, (18), pp. 2162-2170

[5] Ding, Z.: 'Consensus output regulation of a class of heterogeneous nonlinear systems', IEEE Trans. Autom. Control, 2013, 58, (10), pp. 2648-2653

[6] Fax, J., Murray, R.: 'Information flow and cooperative control of vehicle formation', IEEE Trans. Autom. Control, 2004, 49, (9), pp. 1465-1476

[7] Olfati-Saber, R., Murray, R.: 'Consensus problems in networks of agents with switching topology and time-delays', IEEE Trans. Autom. Control, 2004, 49, (9), pp. 1520-1533

[8] Ren, W., Beard, R.: 'Consensus seeking in multiagent systems under dynamically changing interaction topologies', IEEE Trans. Autom. Control, 2005, 50, (5), pp. 655-661

[9] Hong, Y., Hu, J., Gao, L.: 'Tracking control for multi-agent consensus with an active leader and variable topology', Automatica, 2006, 42, (7), pp. 1177-1182

[10] Ren, W., Moore, K. L., Chen, Y.: 'High-order and model reference consensus algorithms in cooperative control of multivehicle systems', ASME J. Dyn. Syst. Meas. Control, 2007, 129, (5), pp. 678-688

[11] Li, Z., Duan, Z., Chen, G., Huang, L.: 'Consensus of multi-agent systems and synchronization of complex networks: a unified viewpoint', IEEE Trans. Circuits Syst. I, 2010, 57, (1), pp. 213-224

[12] Ni, W., Cheng, D.: 'Leader-following consensus of multi-agent systems under fixed and switching topologies', Syst. Control Lett., 2010, 59, (3), pp. 209-217

[13] Ding, Z.: 'Consensus disturbance rejection with disturbance observers', IEEE Trans. Ind. Electron., 2015, 62, (9), pp. $5829-5837$

[14] Yu, W., Chen, G., Lu, J., Kurths, J.: 'Synchronization via pinning control on general complex networks', SIAM J. Contr. Optim., 2013, 51, (2), pp. 1395-1416

[15] Li, Z., Ren, W., Liu, X., Fu, M.: 'Consensus of multi-agent systems with general linear and Lipschitz nonlinear dynamics using distributed adaptive protocols', IEEE Trans. Autom. Control, 2013, 58, (7), pp. 1786-1791

[16] Liu, K., Xie, G., Ren, W., Wang. L.: 'Consensus for multi-agent systems with inherent nonlinear dynamics under directed topologies', Syst. Control Lett., 2013, 62, (2), pp. 152-162

[17] Ding, Z.: 'Adaptive consensus output regulation of a class of nonlinear systems with unknown high-frequency gain', Automatica, 2015, 51, (1), pp. 348-355

[18] Lin, P., Jia, Y., Li, L.: 'Distributed robust $H_{\infty}$ consensus control in directed networks of agents with time-delay', Syst. Control Lett., 2008, 57, (8), pp. 643-653

[19] Lin, P., Jia, Y.: 'Robust $H_{\infty}$ consensus analysis of a class of second-order multi-agent systems with uncertainty', IET Control Theory Appl., 2010, 4, (3), pp. 487-498 
[20] Li, Z., Duan, Z., Chen, G.: 'On $H_{\infty}$ and $H_{2}$ performance regions of multi-agent systems', Automatica, 2011, 47, (4), pp. 793-803

[21] Liu, Y., Jia, Y.: ' $H_{\infty}$ consensus control for multi-agent systems with linear coupling dynamics and communication delays', Int. J. Syst. Sci., 2012, 43, (1), pp. 50-62

[22] Wang, J., Duan, Z., Li Z., Wen, G.: 'Distributed $H_{\infty}$ and $H_{2}$ consensus control in directed networks', IET Control Theory Appl., 2013, 8, (3), pp. 193-201

[23] Wen, G., Hu, G., Yu, W., Chen, G.: 'Distributed $H_{\infty}$ consensus of higher order multiagent systems with switching topologies', IEEE Trans. Circuits Syst. II, 2014, 61, (5), pp. 359-363

[24] Saboori, I., Khorasani, K.: ' $H_{\infty}$ consensus achievement of multi-agent systems with directed and switching topology networks', IEEE Trans. Autom. Control, 2014, 59, (11), pp. 3104-3109

[25] Li, Z., Liu, X., Fu, M., Xie, L.: 'Global $H_{\infty}$ consensus of multi-agent systems with Lipschitz non-linear dynamics', IET Control Theory Appl., 2012, 6, (13), pp. 2041-2048

[26] Wen, G., Yu, W., Chen, M. Z., Yu, X., Chen, G.: ' $H_{\infty}$ Pinning synchronization of directed networks with aperiodic sampled-data communications', IEEE Trans. Circuits Syst. I, 2014, 61, (11), pp. 3245-3255

[27] Li, Z., Duan, Z.: 'Cooperative Control of Multi-Agent Systems: A Consensus Region Approach' (CRC Press, Boca Raton, 2014)

[28] Smith, O.: 'A controller to overcome dead time', ISA, 1959, 6, (2), pp. 28-33

[29] Palmor, Z.: 'Modified predictors', in Levine, W. (Ed.) : 'The Control Handbook' (CRC Press, 1996), Section 10.9

[30] Manitius, A., Olbrot, A.: 'Finite spectrum assignment problem for systems with delays', IEEE Trans. Autom. Control, 1979, 24, (2), pp. 541-552

[31] Kwon, W., Pearson, A.: 'Feedback stabilization of linear systems with delayed control', IEEE Trans. Autom. Control, 1980, 25, (2), pp. 266-269

[32] Artstein, Z.: 'Linear systems with delayed controls: a reduction', IEEE Trans. Autom. Control, 1982, 27, (4), pp. 869-879

[33] Engelborghs, K., Dambrine, M., Roose, D.: 'Limitations of a class of stabilization methods for delay systems', IEEE Trans. Autom. Control, 2001, 46, (2), pp. 336-339

[34] Zhou, B., Lin, Z., Duan, G.: 'Truncated predictor feedback for linear systems with long time-varying input delays', Automatica, 2012, 48, (10), pp. 2387-2399

[35] Lin, Z., Fang, H.: 'On asymptotic stability of linear systems with delayed input', IEEE Trans. Autom. Control, 2007, 52, (6). pp. 998-1013

[36] Zhou, B., Lin, Z., Duan, G.: 'Stabilization of linear systems with input delay and saturation-a parametric Lyapunov equation approach', Int. J. Robust Nonlinear Control, 2010, 20, (13), pp. 1502-1519

[37] Yoon, S. Y., Lin, Z.: 'Truncated predictor feedback control for exponentially unstable linear systems with time-varying input delay', Syst. Control Lett., 2013, 62, (10), pp. 837-844

[38] Ding, Z., Lin, Z.: 'Truncated state prediction for control of Lipschitz nonlinear systems with input delay', Proc. 53rd IEEE Conf. Decision Control, 2014, pp. 1966-1971

[39] Zhou, Bin.: 'Consensus of delayed multi-agent systems by reduced-order observer-based truncated predictor feedback protocols', IET Control Theory Appl., 2014, 8, (16), pp. 1741-1751

[40] Zhou, B., Lin, Z.: 'Consensus of high-order multi-agent systems with large input and communication delays', Automatica, 2014, 50, (2), pp. 452-464

[41] Ding, Z.: 'Consensus control of a class of Lipschitz nonlinear systems', Int. J. Control, 2014, 87, (11), pp. 2372-2382 
[42] Gu, K., Kharitonov, V. K., Chen, J.: 'Stability of Time-Delay Systems’ (Birkhauser: Basel, 2003)

[43] Yoon, S. Y., Anantachaisilp, P., Lin, Z.: 'An LMI approach to the control of exponentially unstable systems with input time delay', Proc. 52nd IEEE Conf. Decision Control, 2013, pp. 312-317 